\title{
Structure of photoluminescence DL-spectra and phase transformation in lightly doped SiC crystals and films
}

\author{
S.I. Vlaskina ${ }^{1,2}$, G.N. Mishinova ${ }^{3}$, V.I. Vlaskin ${ }^{4}$, V.E. Rodionov ${ }^{2}$, G.S. Svechnikov ${ }^{2}$ \\ ${ }^{1}$ Yeoju Institute of Technology (Yeoju University), \\ 338 Sejong-ro, Yeoju-eup, Yeoju-gun, Gyeonggi-do, 469-705 Korea \\ ${ }^{2} V$. Lashkaryov Institute of Semiconductor Physics, National Academy of Sciences of Ukraine, \\ 41, prospect Nauky, 03028 Kyiv, Ukraine; e-mail: businkaa@mail.ru \\ ${ }^{3}$ Taras Shevchenko Kyiv National University, \\ 64, Volodymyrs'ka str., 01033 Kyiv, Ukraine \\ ${ }^{4}$ Sensartech, 2540 Lobelia Dr., Oxnard, 93036 California, USA
}

\begin{abstract}
In this work, the results of investigations of $\mathrm{DL}_{i}$ spectra in $\alpha$-SiC crystals and films with a low impurity concentration have been presented. Photoluminescence spectra of lightly doped $\mathrm{SiC}$ single crystals and films with the impurity concentration of $N_{D}-N_{A}$ $(2 \ldots 8) \cdot 10^{16} \mathrm{~cm}^{-3}, N_{D} \sim(5 \ldots 8) \cdot 10^{17} \mathrm{~cm}^{-3}$, and $N_{D}-N_{A}>3 \cdot 10^{17} \mathrm{~cm}^{-3}, N_{D} \geq 1 \cdot 10^{18} \mathrm{~cm}^{-3}\left(N_{\mathrm{DL}^{-}}\right.$ samples) were investigated within the temperature range $4.2 \ldots 77 \mathrm{~K}$. Complex spectroscopic study of one-dimensional disordered structures caused by solid phase transformations in $\mathrm{SiC}$ crystals was presented. Disordered growth D-layers in lightly doped crystals and $\alpha$-SiC films were investigated using low temperature photoluminescence. The analysis testifies that DL and SF spectra hand-in-hand follow the structure transformations. It has been shown that the DL and SF spectra of luminescence reflect the fundamental logic of $\mathrm{SiC}$ polytypes structure. This allows to observe the structure changes at the phase transformations, the growth of SiC polytypes and to control their aggregates.
\end{abstract}

Keywords: silicon carbide, polytype, stacking fault, photoluminescence spectra, phase transitions.

Manuscript received 03.12.14; revised version received 18.03.15; accepted for publication 27.03.15; published online 08.06.15.

\section{Introduction}

The study of polytypism in semiconductors crystals has a long history [1-4]. Nevertheless, the question about the mechanism of their structure reconstruction is still open. Most of these investigations was carried out using X-ray diffraction (XRD) and high resolution transmission electron microscopy (HREM). The evaluation of relation between the crystal structure and optical properties (photoluminescence (PL), absorption) is widely studied using the materials possessing polytypism.

Silicon carbide is the best candidate for studying polytypism in semiconductors. The real structure, vacancies and dislocations, accompanied by the lattice vibration, properties of the polytype layers and their interactions can be obtained from the low-temperature optical spectra. The mechanism of the solid phase transformation between cubic and hexagonal phases is of great importance in relation with determination of the dominant role of the kinetic and thermodynamic factors in stabilization of certain polytypes. Low-temperature photoluminescence (LTPL) spectra at different registration conditions and temperature parameters together with the parallel controlling the phase and structure disorder by using X-ray and electron diffraction methods are capable to reveal new important 
information about interphase transformation at the level of electron states in the crystal [2,3]. Along with other factors responsible for the polytypism energetics (at the atomic level), the latter play a dominant role in stabilization of certain polytypes as well as in interphase transformation.

The low energy of stacking faults in $\mathrm{SiC}$ facilitates structure transformations in these crystals. The magnitude of this energy depends on the impurity concentration (in doped crystals it is higher due to impurity adsorption on the stacking faults) and temperature. Within the temperature range of phase transformations $(1900 \ldots 2100){ }^{\circ} \mathrm{C}$ in pure crystals with minimum impurity concentrations of $\left(\leq 1 \cdot 10^{17} \mathrm{~cm}^{-3}\right)$ the stacking faults energy is close to $(0.5 \ldots 2) \mathrm{mJ} / \mathrm{m}^{2}$. Equilibrium configurations are determined using minimization of the total energy. It was shown that the energy difference due to atomic relaxation at the stacking domains does not exceed $1 \mathrm{eV}$. Therefore, it couldn't sustain energetic stability of the given modification. Spectroscopy of the stacking faults and their detailed structure in the high purity SiC crystals was described in $[5,6]$.

Defects in $\mathrm{SiC}$ crystals with a low level of impurities (with in-grown original defects) have been investigated in $\operatorname{Ref}[6,7]$.

The spectra of $\mathrm{DL}_{i}$ type are different from the SFi spectra and have other principles of construction and behavior. The $\mathrm{DL}_{i}$ spectra are related to the broad donoracceptor paires (DAP) emission band but all of them have identical behavior. The data from the photoluminescence, excitation and absorption spectra show similarity of different $\mathrm{DL}_{i}$ spectra. However, the structure of SF spectra was discussed in details $[5,6]$. $\mathrm{DL}_{i}$ spectra are still under investigation. In this paper, investigation of $\mathrm{DL}_{i}$ spectra in $\alpha$-SiC crystals and films with a low impurity concentration have been presented.

\section{Experiment}

The group of single crystalline $\alpha$-SiC crystals grown by Lely's method, and films grown by Tairov's and CVD method were selected for the research. They were initially different in the phase composition ( $\alpha$ or $\beta$ phase) as well as in the structural disorder.

The X-ray (Laue's method) and electron diffraction methods were used to detect symmetry and layer parameters of the crystals. Moreover, in some cases to analyze structural peculiarity of the allocated blocks the transmission electron microscopy technique (TEM) $(\times 15000)$ was used.

Complex spectroscopic study of one-dimensional disordered structures due to the solid phase transformations in SiC crystals was performed.

Disordered growth of D-layers in lightly doped crystals and $\alpha$-SiC films was investigated using lowtemperature photoluminescence.
In the PL experiments, nitrogen LGI-21 operating at the wavelength $337 \mathrm{~nm}(3.68 \mathrm{eV})$, helium-cadmium LG-70 - $441.6 \mathrm{~nm}(2.807 \mathrm{eV})$ and Ar-lasers $\lambda=$ $488 \mathrm{~nm}, h v=2.54 \mathrm{eV}$, respectively, were used. Also, mercury ultrahigh pressure lamp SVDSh-1000 with the UV-2 filter, and xenon lamp DKSSh-1000 were used.

PL spectra of lightly doped SiC single crystals and films with the impurity concentration of $N_{D}-N_{A} \sim$ $(2 \ldots 8) \cdot 10^{16} \mathrm{~cm}^{-3}, N_{D} \sim(5 \ldots 8) \cdot 10^{17} \mathrm{~cm}^{-3}$, and $N_{D}-N_{A}$ $>3 \cdot 10^{17} \mathrm{~cm}^{-3}, N_{D} \geq 1 \cdot 10^{18} \mathrm{~cm}^{-3}\left(\mathrm{~N}_{\mathrm{DL}}\right.$-samples $)$ were investigated within the temperature range 4.2 to $77 \mathrm{~K}$.

\section{Results and discussion}

The $\mathrm{DL}_{i}$ spectra are prevailing as compared to the background of broad DAP spectral band as it was depicted in Refs. [5, 6] (Fig. 1).

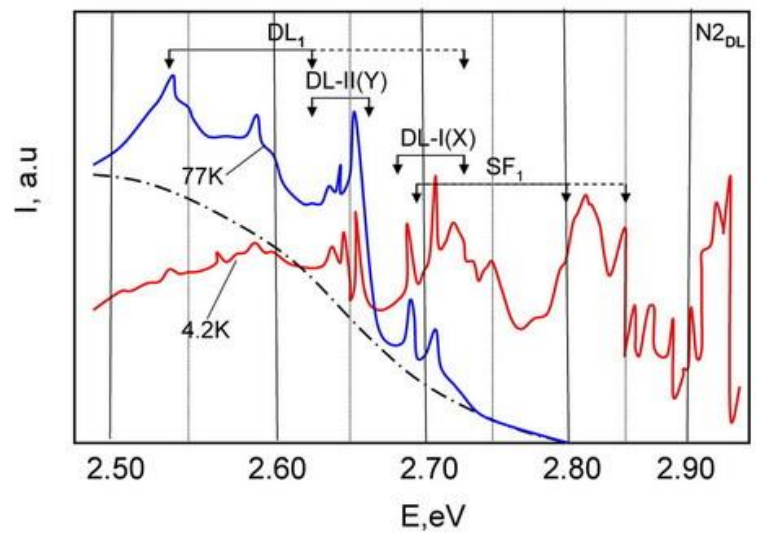

Fig. 1. DL-type spectra of crystals (at $4.2 \mathrm{~K}$ and $77 \mathrm{~K}$ ) on the background of a broad structureless band. $\mathrm{N} 22_{\mathrm{DL}}-$ $3 \cdot 10^{16} \mathrm{~cm}^{-3}$.

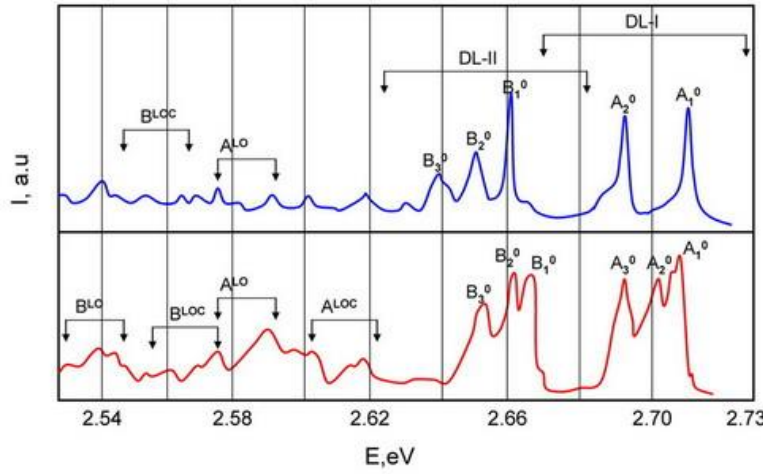

Fig. 2. Structure of the $\mathrm{DL}_{1}$ spectrum. Phonons of extended center Brillouin zone (LO 119...120 meV) and local phonon (LOC $91 \mathrm{meV}$ ) are involved. The fine structure of the zerophonon part of spectrum can be separated into two groups (DL-I and DL-II) for the samples $\mathrm{N} 1_{\mathrm{DL}}-2 \cdot 10^{16} \mathrm{~cm}^{-3}, \mathrm{~N} 2 \mathrm{DL}$ $-3 \cdot 10^{16} \mathrm{~cm}^{-3}$. 

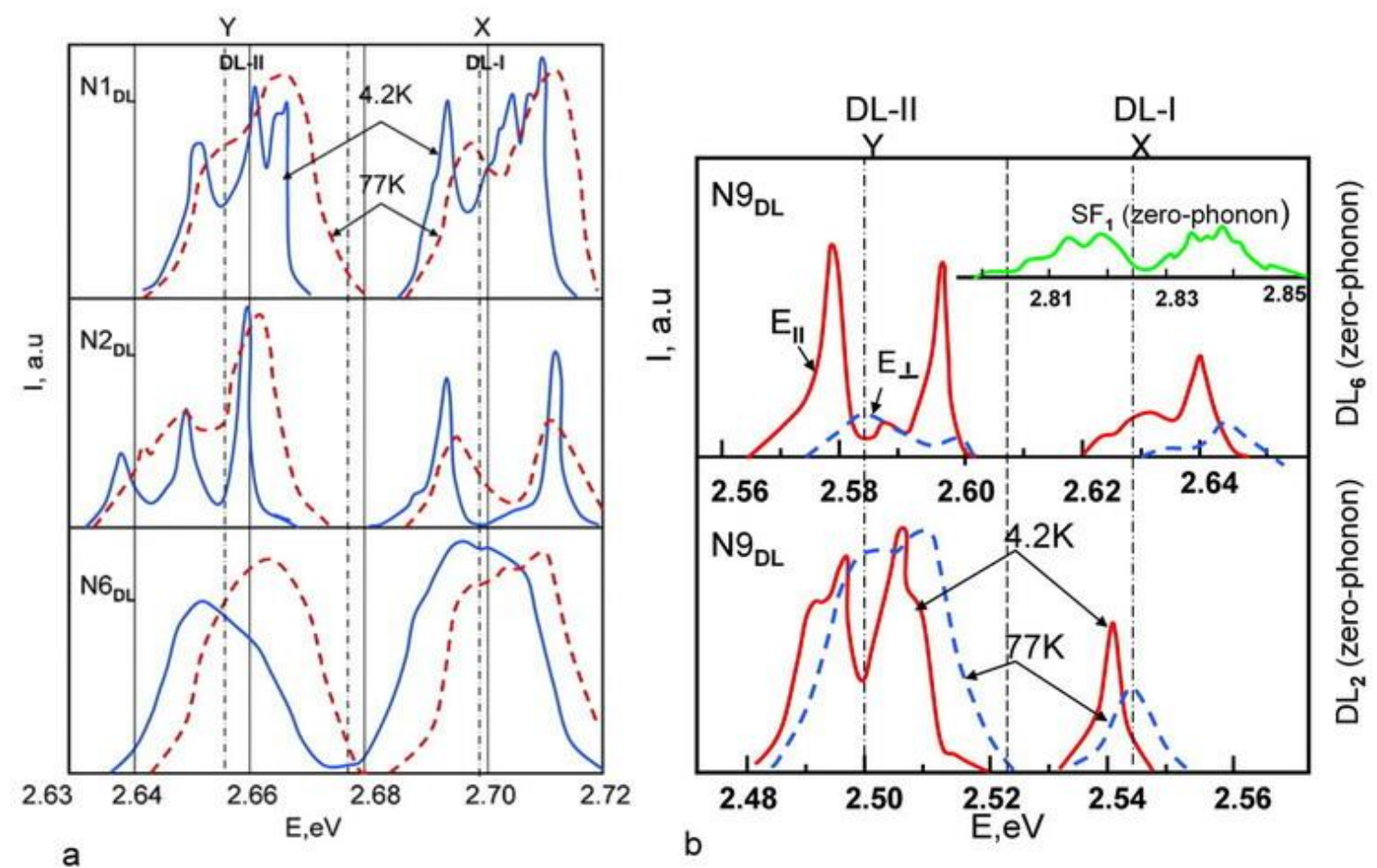

Fig. 3. The fine structure of the DL spectra in the samples with different contents of impurities. $\mathrm{N} 1_{\mathrm{DL}}-2 \cdot 10^{16} \mathrm{~cm}^{-3}, \mathrm{~N} 2_{\mathrm{DL}}-$ $3 \cdot 10^{16} \mathrm{~cm}^{-3}, \mathrm{~N} 6_{\mathrm{DL}}-7 \cdot 10^{17} \mathrm{~cm}^{-3}, \mathrm{~N} 9_{\mathrm{DL}}-7 \cdot 10^{16} \mathrm{~cm}^{-3}$.

a

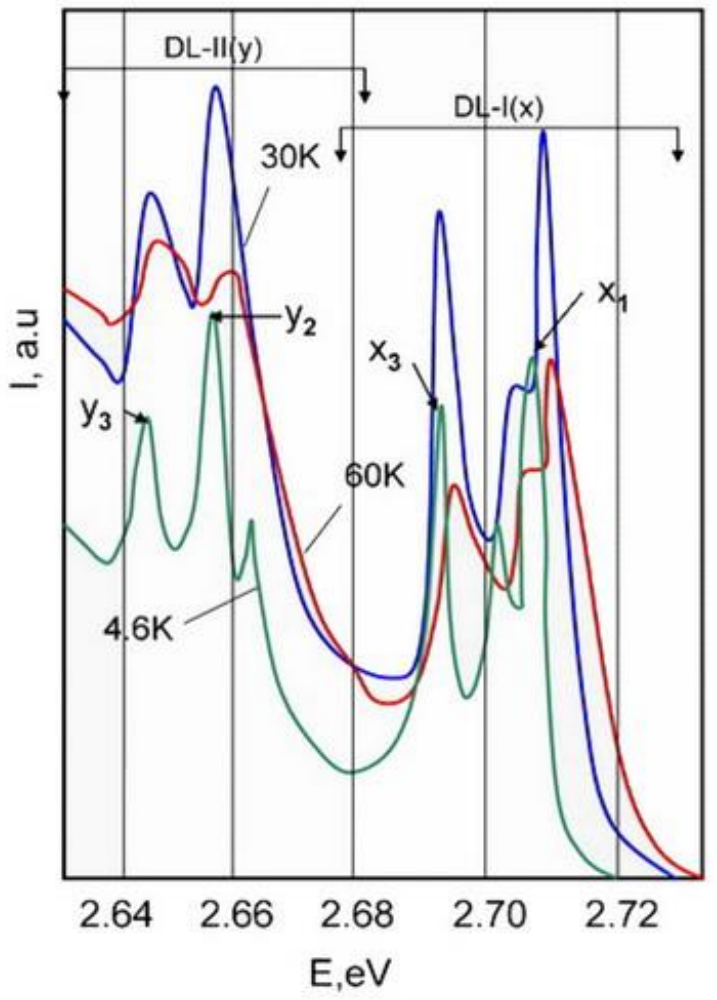

b
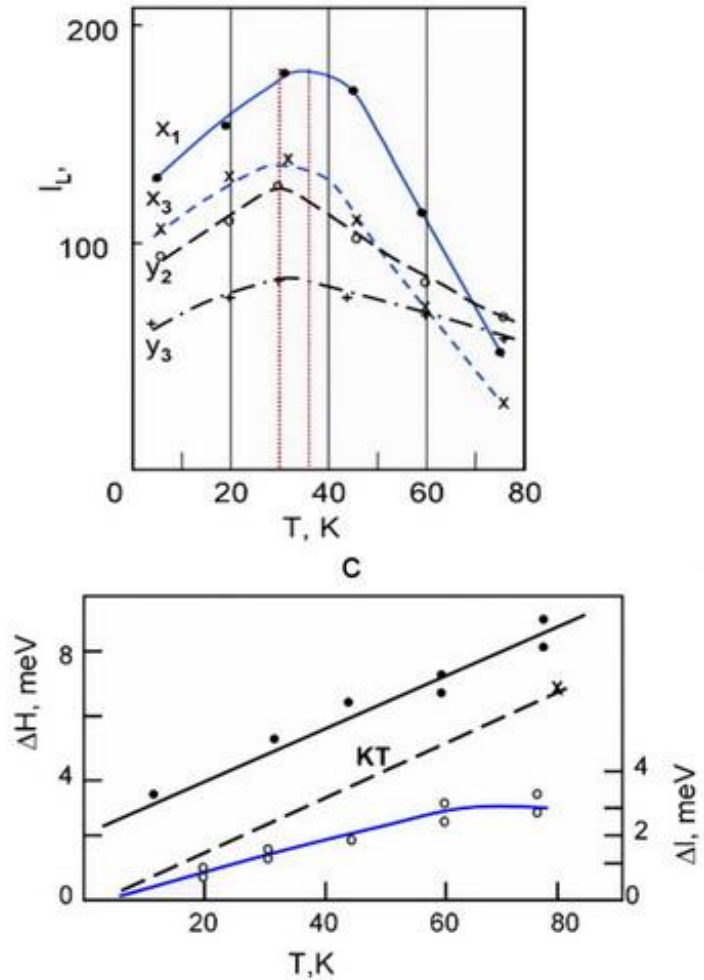

Fig. 4. Dependence of the fine structure of the zero-phonon spectra behavior on temperature for the sample $\mathrm{N} 1_{\mathrm{DL}}$. a) Dependence of the fine structure DL-I and DL-II spectra on temperature; b) temperature dependence of certain peaks (see in Fig. 4a) of fine structure; c) temperature broadening and energy shift for the elements of thin structure. 
The data of all the $\mathrm{DL}_{i}$ spectra for different samples and in different registration conditions show that the $\mathrm{DL}_{i}$ spectrum consists of two zero-phonon parts - two linear groups $\mathrm{X}$ and $\mathrm{Y}$, and of their phonon replicas. The samples with the minimum concentration $N_{D}-N_{A}$ demonstrated well defined line structure (with good resolution) $\mathrm{X}$ and $\mathrm{Y}$, which allows accurate decoding the spectrum.

In the SF spectra, the zero-phonon part was absent (it was calculated and depicted in Fig. 1 with dash line) and only the spectra of the phonon replica can be seen. On the contrary, in the DL spectra both the zero-phonon part and phonon replica were present. As well as for the SF spectra [6], the fine structure of the zero-phonon part can be separated into two parts DL-I and DL-II. Decoding the DL spectrum shows that it comprises zerophonon parts - two linear group $\mathrm{X}$ and $\mathrm{Y}$ and their phonon replicas as one with LA, LO and local phonons with the energies $(70 \pm 1.0),(119 \pm 1.0)$ and, $(91 \pm 1.0) \mathrm{meV}$, respectively. LO phonon is the most active in forming the spectrum.

The structure of DL-I spectrum is shown in Fig. 2. It includes phonons of central expanded Brillouin zone (LO 119...120 meV) and local phonon (LOC $91 \mathrm{meV}$ ).

Not to confound with the SF spectra (the fine structure of the SF spectra is given in Ref. [6]), we denominate them DL-I(X) and DL-II(Y). The fine structure of the DL spectra visible in the samples with different levels of impurities is shown in Fig. 3. The fine structure is the function of the temperature and polarization of the excitation light.

Dependence of the fine structure of the zerophonon spectra behavior via temperature for the sample $\mathrm{N} 1_{\mathrm{DL}}$ is shown in Fig. 4. With the temperature increase, the spectral position, halfwidth and intensity of the elements of the DL spectrum vary.

Considering the temperature dependence of all the DL spectra, it is clear that they have the same structure logic: zero-phonon parts - two linear group $\mathrm{X}$ and $\mathrm{Y}$ and their phonon replicas as that with LA, LO and local phonon (at $4.2 \mathrm{~K}$ ) participation.

Moreover, the behavior of each DL spectra under different registration conditions is identical. In the temperature interval of 4.6 to $77 \mathrm{~K}$, each element of the fine structure $\mathrm{X}$ and $\mathrm{Y}$ groups shifts towards shorter wavelength range and broadens. Intensity is changed reaching a peak, following by the quick quench (Fig. 4b).

The intensity first rises, peaking at $\sim 30 \mathrm{~K}$, and then returns back to the initial level (as at $4.2 \mathrm{~K}$ ) approximately at $60 \mathrm{~K}$. The intensity of the phonon part of the spectrum as to zero-phonon part rises. At $60 \mathrm{~K}$, there is no phonon replica bound up to the local phonon (Fig. 4b).

With a further temperature rise, the intensity of the DL spectrum continues to fall and blurred $\mathrm{X}$ and $\mathrm{Y}$ groups lower their intensity in different ways without any energy shift. The same temperature behavior of the
$\mathrm{DL}_{i}$ spectra points out to the identity of the centers responsible for DL luminescence.

Behavior of the $\mathrm{DL}_{i}$ spectra significantly differs from that of the SF spectra. The zero-phonon part is completely absent in the SF spectra. From the zerophonon part of the spectrum, one can see that each line of the fine structure has a maximum within the temperature range $30 \ldots 35 \mathrm{~K}$. Above the temperature $60 \mathrm{~K}$, local phonons disappear. It is possible that there is carriers pumping from the shallow levels near the bottom of the exciton conduction band. In the region of $60 \mathrm{~K}$, these sources deplete. Each of the line in the fine structure broadens accordingly to linear dependence within the temperature range 4.6 to $60 \mathrm{~K}$.

On the contrary, the SF spectra within the temperature range $30 \ldots 35 \mathrm{~K}$ completely disappear. The same spectra can be detected at the reverse phase transition of cubic to hexagonal $\mathrm{SiC}[8,9]$.

Like to SF, the DL spectra comprise two parts superposing each other. Here, the energy width of the SF spectrum is equal to the width of each part in the DL spectrum.

$\mathrm{X}$ and $\mathrm{Y}$ components of the zero-phonon parts of DL spectra photoluminescence $(T=4.2 \mathrm{~K}$ and $77 \mathrm{~K})$ and phosphorescence $(t=3 \mathrm{~ms}, T=4.2 \mathrm{~K})$ for the DL samples with different concentrations $N_{D}-N_{A}$ are shown in Fig. 3. All the DL spectra demonstrate the same behavior of the fine structure $\mathrm{X}$ and $\mathrm{Y}$ groups irrespective of DL-I or DL-II affiliation. They are characterized by the same energy width, temperature broadening and shift to the shorter wavelength range.

With the increase of the concentration of noncompensated impurity $N_{D}-N_{A}$ from $2 \cdot 10^{16} \mathrm{~cm}^{-3}$ to $(5 \ldots 7) \cdot 10^{17} \mathrm{~cm}^{-3}$, one can see broadening of the spectra structure and the fine structure of $\mathrm{X}, \mathrm{Y}$ components faded (blurred) even at $T=4.2 \mathrm{~K}$ (sample $\mathrm{N} 9_{\mathrm{DL}}$, Fig. 3b). Here, temperature $(4.2 \ldots 77 \mathrm{~K})$ band shift is similar to that observed.

Evaluation of the energy position of DL and SF spectra has shown that the region of the phase transition consists of thin lamellas $(10 \ldots 100 \AA)$ comprising the set of twinned $\beta$-phase plates, and such base forms of $\alpha$ SiC as: $15 \mathrm{R}\langle 23\rangle(a=12.5 \AA), 6 \mathrm{H}\langle 33\rangle(a=15 \AA), 21 \mathrm{R}$ $\langle 34\rangle(a=17.5 \AA), 8 \mathrm{H}\langle 44\rangle(a=20 \AA), 10 \mathrm{H}\langle 55\rangle(a=$ $25 \AA)$ and $14 \mathrm{H}\langle 77\rangle(a=35 \AA)$.

Table 1. Band gap (optical $E_{g}$ and exciton $E_{g x}$ ) in SiC.

\begin{tabular}{|c|c|c|c|}
\hline Polytype & $E_{g}$ & $E_{g x}$ & $\Delta=E_{g}-E_{g x}$ \\
\hline $6 \mathrm{H}$ & 3.109 & 3.024 & 0.085 \\
\hline $33 \mathrm{R}$ & 3.087 & 3.002 & 0.085 \\
\hline $21 \mathrm{R}$ & 2.938 & 2.853 & 0.085 \\
\hline $8 \mathrm{H}$ & 2.868 & 2.783 & 0.085 \\
\hline $10 \mathrm{H}_{2}$ & 2.798 & 2.853 & 0.085 \\
\hline $14 \mathrm{H}_{2}$ & 2.698 & 2.613 & 0.085 \\
\hline
\end{tabular}


Table 2. Difference between the position of the center of symmetry in the zero-phonon part of SF spectra (normalized to $E_{g x}$ ) and the position of the center of symmetry of DL-I (X) (data from Refs. [5-7]).

\begin{tabular}{|c|c|}
\hline Polytype & $\mathrm{SF}^{E g x}-\mathrm{DL}-\mathrm{I}(\mathrm{X})=\Delta_{1}$ \\
\hline $21 \mathrm{R}$ & $2.823-2.698=0.125$ \\
\hline $10 \mathrm{H}_{2}$ & $2.683-2.558=0.125$ \\
\hline $14 \mathrm{H}_{2}$ & $2.583-2.458=0.125$ \\
\hline
\end{tabular}

Table 3. Difference between the position of the center of symmetry in the zero-phonon part of SF spectra (normalized to $E_{g x}$ ) and the position of the center of symmetry in DL-II (Y) (data from Ref. [5-7].

Shift between centers $\Delta_{1}-\Delta_{2}=0.043 \mathrm{eV}$.

\begin{tabular}{|c|c|}
\hline Polytype & $\mathrm{SF}^{E g x}-\mathrm{DL}-\mathrm{II}(\mathrm{Y})=\Delta_{2}$ \\
\hline $21 \mathrm{R}$ & $2.823-2.655=0.168$ \\
\hline $10 \mathrm{H}_{2}$ & $2.683-2.515=0.168$ \\
\hline $14 \mathrm{H}_{2}$ & $2.583-2.415=0.168$ \\
\hline
\end{tabular}

Table 4. Correlation between the centers of symmetry in the zero-phonon parts of the SF and DL spectra.

\begin{tabular}{|c|l|}
\hline Polytype & $\mathrm{DL}-\mathrm{II}(\mathrm{Y})+E_{a}=\mathrm{SF}^{E g}+0.03=E_{g}$ \\
\hline $21 \mathrm{R}$ & $2.655+0.253=2.908+0.03=2.938$ \\
\hline $10 \mathrm{H}_{2}$ & $2.515+0.253=2.768+0.03=2.798$ \\
\hline $14 \mathrm{H}_{2}$ & $2.415+0.253=2.668+0.03=2.698$ \\
\hline
\end{tabular}

Here: $\mathrm{DL}-\mathrm{II}(\mathrm{Y})+E_{a}=\mathrm{SF}^{E g}+0.03=E_{g} ; E_{a}-$ acceptor that forms DL-I(X) and DL-II(Y) spectra.

Table 5. Correlation between the difference of the position of the center of symmetry in non-phonon parts of SF (optical and exciton) and band gap $E_{g}$ (optical and exciton).

\begin{tabular}{|c|c|}
\hline Polytype & $\mathrm{SF}^{E g}-\mathrm{SF}^{E g x}=\Delta=E_{g}-E_{g x}$ \\
\hline $21 \mathrm{R}$ & $2.908-2.823=0.085$ \\
\hline $10 \mathrm{H}_{2}$ & $2.798-2.613=0.085$ \\
\hline $14 \mathrm{H}_{2}$ & $2.668-2.583=0.085$ \\
\hline
\end{tabular}

Thus, DL and SF spectra hand-in-hand follow the structure transformations. Like to SF, the DL spectra comprise two parts superposing each other. Here, the energy width of the SF spectrum equals the width of each part of the DL spectrum. If one could draw a line through the middle of superposing parts DL-X, DL-Y and SF-I, SF-II and estimate $E_{g}$ for the different polytypes (Table 1) the intelligible correlation is revealed (Tables 2-5). From the data above, the DL luminescence spectra reflect the fundamental logic of $\mathrm{SiC}$ polytype structure. Owing to it, one can observe the structure changes caused by the phase transformations and growth of $\mathrm{SiC}$ polytypes and their aggregates.

\section{Conclusion}

All DL spectra have identical construction logic: they comprise zero-phonon part - two linear groups $\mathrm{X}$ and $\mathrm{Y}$ and their phonon replicas (as one) with participation of LA, LO and local phonons (4.2 K).

Behavior of each DL spectra under different registration conditions is identical. Within the temperature interval $4.6 \ldots 77 \mathrm{~K}$, each element of the fine structure $\mathrm{X}$ and $\mathrm{Y}$ groups shifts towards shorter wavelength range and broadens. Intensity is changing reaching a peak, following by the quick quench. The DL and SF spectra kind of together watch the structure 
transformations. Like to SF, the DL spectra comprise two parts superposing each other. Here, the energy width of the SF spectrum equals the width of each part of the DL spectrum. The luminescence DL and SF spectra reflect the fundamental logic of $\mathrm{SiC}$ polytype structure. It allows observation of the structure changes at the phase transformations, the growth of SiC polytypes and control their aggregates.

\section{References}

1. P.R. Van Loan, A study of polytypism in silicon carbide. Research Department Norton Company, Chippawa, Ontario, Canailo // The American Mineralogist, 52, July-August, p. 946-956 (1967).

2. Fei Yan, Dissertation "Low Temperature Study on Defect Centers in Silicon Carbide", University of Pittsburgh, 2009 (Dissertation LTPL-Choyke Pittsburg, 2009 pdf).

3. W.J. Choyke, H. Matsunami, SiliconCarbide: Recent Major Advances // books.google.co.kr/books?isbn =3540404589 Gerhard Pensl - 2004 .

4. Kazuaki Kobayashi, Shojiro Komatsu, First principle study of $12 \mathrm{H}-$, and $18 \mathrm{H}-\mathrm{SiC}$ polytypes // J. Phys. Soc. Jpn. Appl. 024714-024714-13 (2012).
5. S.I. Vlaskina, G.N. Mishinova, V.I. Vlaskin, V.E. Rodionov, G.S. Svechnikov, 8H-, 10H-, 14H-SiC formation in $6 \mathrm{H}-3 \mathrm{C}$ silicon carbide phase transitions // Semiconductor Physics, Quantum Electronics and Optoelectronics, 16(3), p. 272-278 (2013).

6. S.I. Vlaskina, G.N. Mishinova, V.I. Rodionov, G.S. Svechnikov, Peculiarity of phase transformations of $\mathrm{SiC}$ crystals and thin films with in-grown original defects // Semiconductor Physics, Quantum Electronics and Optoelectronics, 17(4), p. 380-383 (2014).

7. S.I. Vlaskina, G.N. Mishinova, L.V. Vlaskin, V.E. Rodionov, G.S. Svechnikov, Nanostructures in lightly doped silicon carbide crystals with polytypic defects // Semiconductor Physics, Quantum Electronics and Optoelectronics, 17(2), p. 155-159 (2014).

8. S.I. Vlaskina, G.N. Mishinova, V.I. Vlaskin, V.E. Rodionov, G.S. Svechnikov, 3C-6H transformation in heated cubic Silicon Carbide 3C-SiC // Semiconductor Physics, Quantum Electronics and Optoelectronics, 14(4), p. $432-437$ (2011).

9. S.I. Vlaskina, G.N. Mishinova, V.I. Vlaskin, V.E. Rodionov, G.S. Svechnikov, S.W. Lee, Silicon carbide phase transition in as-grown 3C-H-polytype junction // Semiconductor Physics, Quantum Electronics and Optoelectronics, 16(3) p. 132-136 (2013). 